\title{
A ASSOCIAÇÃO DO CETUXIMABE NA TERAPIA DO CÂNCER COLORRETAL: UMA REVISÃO DA LITERATURA ${ }^{1}$
}

\author{
THE ASSOCIATION OF CETUXIMABE IN \\ COLORRETAL CANCER THERAPY: A LITERATURE REVIEW
}

\section{Bruna Donato Gularte ${ }^{2}$, Marcel Henrique Marcondes Sari ${ }^{3}$ e Luana Mota Ferreira ${ }^{4}$}

\section{RESUMO}

O câncer colorretal é um dos tipos de câncer mais incidentes no Brasil. Nos últimos anos, as taxas de mortalidade reduziram devido aos avanços terapêuticos, principalmente pelo uso de anticorpos monoclonais como o cetuximabe. Nesse contexto, este estudo consiste em uma revisão integrativa da literatura, realizada a partir do levantamento de dados relacionados ao efeito da associação do cetuximabe na terapia do câncer colorretal. Foram utilizadas as bases de dados Web of Science, PubMed e Scopus, avaliando as publicações no período de 2015 até 2020. Utilizou-se os descritores: "Cetuximabe" e "câncer colorretal" e "FOLFOX" ou "FOLFIRI", nos idiomas português e inglês. A busca resultou em 539 artigos científicos dos quais 12 publicações foram selecionadas para discussão após a aplicação dos critérios de inclusão e exclusão. Os resultados mostram que $50 \%$ das publicações tratam da inserção do cetuximabe no protocolo FOLFOX (6), 33,3\% no protocolo FOLFIRI (4) e 16,66\% trazem ambos os protocolos em comparação ao cetuximabe (2). De forma geral, o cetuximabe melhora as taxas de sobrevida livre de progressão, sobrevida global e resposta objetiva principalmente dos pacientes sem mutações no gene KRAS. Os medicamentos biológicos têm ganhado espaço na oncologia por ser uma terapia-alvo, melhorando as taxas de sobrevida e redução da incidência de eventos adversos, os quais são relacionados às reações infusionais. Esta revisão integrativa demonstra os benefícios do uso do um anticorpo monoclonal cetuximabe no câncer de colorretal, fornecendo um material informativo de fácil leitura, que colabora com a tomada de decisão terapêutica, para um tratamento seguro e eficaz para os pacientes.

Palavras-chave: Anticorpo monoclonal, terapia alvo, anti-EGFR, tumores gastrointestinais.

\section{ABTRACT}

Colorectal cancer is one of the most common types of cancer in Brazil. Recently, the mortality rates have been reducing due to the therapeutic advances achieved, due to the development of monoclonal antibodies, such as cetuximab. This review aimed to evaluate the benefit of cetuximab associated with protocols used in colorectal cancer treatment. The study is an integrative literature review, which was carried out using Web of Science, PubMed and Scopus databases, within the period of 2015 to 2020. The descriptors "Cetuximab" and "colorectal cancer" and "FOLFOX" or "FOLFIRI" were used in Portuguese and English languages. The preliminary screening selected 539 scientific articles, which were reduced to 12 after applying the inclusion and exclusion criteria. The results showed that $50 \%$ of the publications approached cetuximab insertion in the FOLFOX protocol (6), 33.3\% in the FOLFIRI protocol (4), while cetuximab in comparison to both FOLFOX 1 Trabalho de Conclusão de Curso.

2 Acadêmica do curso de Farmácia, Universidade Federal de Santa Maria, Santa Maria, Brasil. E-mail: brunadonato2008@ hotmail.com ORCID:https://orcid.org/0000-0002-2743-1797

3 Doutor, Curso de Farmácia, Instituto de Desenvolvimento Educacional de Passo Fundo - UNIDEAU, Passo Fundo, Brasil. E-mail: marcelsari@ideau.com.br ORCID: https://orcid.org/0000-0002-9913-9306

4 Doutora, Programa de Pós-graduação em Ciências Farmacêuticas, Centro de Ciências da Saúde, Universidade Federal de Santa Maria, Santa Maria, Brasil. E-mail: luana.ferreira@ufsm.br. ORCID:https://orcid.org/0000-0001-9951-587X 
and FOLFIRI was reported in $16.66 \%$ of the studies (2). The studies showed that cetuximab improved the overall survival rates, mainly in patients without mutations in the KRAS gene. Such a class of drugs has gained space in the oncology field as it is a targeted therapy, which improves survival rates and reduces the incidence of adverse events, which are related to infusion reactions. This integrative review demonstrates the benefits of using a monoclonal antibody in colorectal cancer therapy. Review studies are easy-to-read informational materials that collaborate with therapeutic decision-making and for safe and effective treatment for patients.

Keywords: Monoclonal antibody, target therapy, anti-EGFR, gastrointestinal tumors.

\section{INTRODUÇÃO}

As neoplasias estão relacionadas a um grande número de óbitos no país e configuram um problema de saúde pública. Entre os diferentes tipos de câncer, o câncer colorretal (CCR) é um dos mais incidentes no Brasil, perfazendo o terceiro tipo mais frequente entre os homens, o segundo para as mulheres, e o quarto tipo de neoplasia mais frequente no país. Para 2020, o INCA estimou cerca de 40.990 novos casos diagnosticados, sendo 20.520 homens e 20.470 mulheres (INCA, 2019).

O CCR abrange tumores que iniciam na parte do intestino grosso, chamado cólon, e no reto, podendo se estender até o ânus. A maior parte desses tumores inicia a partir de pólipos adenomatosos, que consistem em lesões benignas que podem crescer na parede interna do intestino e, ainda, sofrer malignização com o tempo. O tipo histopatológico mais comum é o adenocarcinoma, sendo uma doença tratável e frequentemente curável quando localizada no intestino sem extensão para outros órgãos por ocasião do diagnóstico (BRASIL, 2014; DEKKER et al., 2019; INCA, 2019).

O desenvolvimento da doença está fortemente associado a fatores de risco hereditários e ambientais como aumento do peso corporal, consumo de carne vermelha e processada em excesso, tabagismo e ingestão excessiva de álcool. Além desses fatores, pacientes com doença inflamatória intestinal como retocolite ulcerativa crônica, doença de Crohn, e aqueles com histórico anterior de câncer colorretal ou adenomas, apresentam maior risco de desenvolver a doença (DEKKER et al., 2019). A incidência do CCR aparece com maior frequência a partir dos 50 anos de idade, porém a doença também pode ocorrer em pessoas com idade inferior a 40 anos, com menor incidência (DE CAMPOS et al., 2017).

A detecção precoce do CCR é importante, pois se detectado em sua fase inicial possibilita maiores chances de tratamento (INCA, 2019). Os tumores de cólon e reto podem ser detectados precocemente por meio de dois exames principais: pesquisa de sangue oculto nas fezes e endoscopias (colonoscopia ou retossigmoidoscopia). Esses exames devem ser realizados principalmente por pessoas com idade superior a 50 anos, com histórico familiar de câncer do intestino grosso ou com sinais e sintomas suspeitos da doença, como sangramento nas fezes, massa (tumoração) abdominal, dor ou desconforto abdominal, alteração do hábito intestinal (diarreia e prisão de ventre alternado), fraqueza e anemia, perda de peso, e alteração na forma das fezes (fezes muito finas e compridas); ou por meio de exames em pessoas sem sinais ou sintomas (rastreamento), mas pertencentes a grupos com maior 
chance de apresentar a doença (SANTOS JR., 2007). O método diagnóstico de escolha para o CCR é a colonoscopia, pois permite a avaliação de todo o intestino grosso e a remoção ou biópsia de pólipos que podem estar localizados fora da área de ressecção da lesão principal (BRASIL, 2014; DEKKER et al., 2019). O tratamento do CCR depende principalmente do tamanho, localização e extensão do tumor. Para a fase inicial é recomendada a cirurgia, com retirada da parte afetada do intestino e nódulos linfáticos próximos a região. Posteriormente, a radioterapia associada ou não à quimioterapia pode ser utilizada para diminuir a possibilidade de recidiva tumoral (BRASIL, 2018; DEKKER et al., 2019).

Os fármacos antineoplásicos são utilizados para destruir as células tumorais e, a combinação de vários agentes terapêuticos com diferentes mecanismos de ação, é uma estratégia eficaz no tratamento do câncer, oferecendo muitos benefícios. Inicialmente, a terapia do CCR era constituída de um único antineoplásico, o 5-fluorouracil (5-FU). Com o passar dos anos, houve a inclusão de outros agentes à terapia, como o ácido fólinico, o irinotecano, a oxaliplatina entre outros, melhorando assim a resposta clínica dos pacientes. A associação de dois ou mais medicamentos com diferentes mecanismos de ação pode atrasar as mutações celulares e o processo de adaptação ao câncer. O efeito sinérgico dos medicamentos, ou seja, a ação combinada dos fármacos leva a um efeito farmacológico potencializado (SAMA; COHEN, 2016; SILVA; CARLOTTO; ROTTA, 2018).

Em casos de metástase, o tratamento paliativo é preconizado para o aumento da sobrevida e da qualidade de vida dos pacientes, exceto em alguns casos de doença metastática exclusivamente hepática. A ressecção curativa de lesões hepáticas pode ser alcançada em pacientes com doença inicialmente ressecável, e até mesmo em situações inicialmente irressecável, devido às novas estratégias que possibilitam a cirurgia de conversão. Com o intuito de melhorar a terapia empregada, através dos avanços na disponibilidade de novos fármacos, com mecanismos de ação distintos e com impacto positivo no tratamento sequencial do CCR, foram descobertos anticorpos monoclonais. Os medicamentos biológicos usados no tratamento do câncer são imunoglobulinas aptas a reconhecer e se ligar a antígenos tumorais específicos desencadeando respostas imunológicas direcionadas, poupando as células saudáveis e provocando efeitos menos tóxicos que a quimioterapia tradicional (ADAM et al., 2009; YE et al., 2015).

Um desses anticorpos monoclonais empregado no tratamento do câncer é o cetuximabe, o qual inibe a proliferação celular e induz a apoptose de células tumorais humanas que expressam o EGFR (Receptor do Fator de Crescimento Epidérmico - Epidermal Growth Factor Receptor). O cetuximabe também inibe a produção de fator angiogênico por células tumorais, bloqueia a migração celular endotelial e provoca uma redução da revascularização tumoral e da metástase (MERCK, 2020). Nesse sentido, pesquisas recentes demonstram que o uso de anticorpos monoclonais e principalmente o cetuximabe tem aumentado a sobrevida dos pacientes com CCR. Dessa forma, o presente estudo buscou analisar a utilização deste medicamento biológico em combinação com os protocolos mais usuais e indicados como primeira linha para o tratamento do câncer colorretal metastático, sendo eles 
o FOLFOX (5-fluororacil, ácido folínico e oxaliplatina) e o FOLFIRI (5-fluorouracil, ácido folínico e irinotecano) através da realização de uma revisão integrativa da literatura.

\section{METODOLOGIA}

O presente estudo trata-se de uma revisão integrativa de literatura, a qual possibilita a busca e análise dos estudos científicos já publicados sobre determinado assunto pré-definido (MENDES; SILVEIRA; GALVÃO, 2008). Para isso, as seguintes etapas foram realizadas: 1) identificação do tema de pesquisa e elaboração da questão norteadora; 2) definição dos critérios de inclusão e exclusão; 3) seleção dos artigos por meio da leitura do título, resumo e, posteriormente, pela leitura na íntegra; 4) coleta das principais informações dos estudos selecionados; 5) análise dos resultados obtidos e, 6) síntese da revisão integrativa (MENDES; SILVEIRA; GALVÃO, 2008).

A questão norteadora para o estudo foi: "Qual o benefício da adição do cetuximabe nos protocolos convencionais empregados no tratamento do CCR?”. Os critérios de inclusão elencados foram os seguintes:

I) estudos que abordam o uso do cetuximabe nos protocolos FOLFOX e/ou FOLFIRI para o tratamento do câncer colorretal;

II) publicações resultantes de artigos originais, ensaios clínicos randomizados e estudos de coorte, com acesso livre, publicados no período de 2015 até 2020. Artigos de revisão foram utilizados para discussão;

III) e estudos divulgados em português e inglês;

Foram excluídas publicações que não respeitassem a delimitação do tema e o objetivo do estudo, artigos de opinião ou reflexão, editoriais e capítulos de livro. O levantamento dos dados foi realizado nas bases de dados Scopus, PubMed e Web of Science. Para a pesquisa nas bases de dados utilizou-se os seguintes descritores: "Cetuximabe" e "câncer colorretal" e "FOLFOX” ou "FOLFIRI", utilizados também no idioma inglês. Na etapa inicial, a seleção dos artigos foi realizada pela leitura do título e do resumo e, após a elegibilidade dos artigos, conforme os critérios de inclusão e exclusão, os trabalhos foram analisados na íntegra. Os principais dados coletados dos artigos foram as taxas de sobrevida livre de progressão (SLP), sobrevida global (SG) e taxa de resposta objetiva (TRO), além da incidência dos principais eventos adversos observados pelos autores.

Os estudos selecionados foram classificados, de acordo com o delineamento metodológico, quanto ao nível de evidência científica, da seguinte maneira: a) nível I: metanálise de múltiplos estudos controlados ou revisões sistemáticas; b) nível II: evidências derivadas de pelo menos um ensaio clínico randomizado, controlado, bem delineado; c) nível III: estudo com delineamento quase-experimental como estudo sem randomização com grupo único pré e pós-teste, séries temporais ou caso-controle; d) nível IV: estudo com delineamento não-experimental como pesquisa descritiva correlacional e 
qualitativa ou estudos de caso e de coorte; e) nível V: relatório de casos ou dado obtido de forma sistemática, de qualidade verificável ou dados de avaliação de programas; f) nível VI: opiniões de autoridades respeitáveis baseada na competência clínica ou opinião de comitês de especialistas, incluindo interpretações de informações não baseadas em pesquisas (MELNYK; FONEOUT-OVERHOLT, 2005).

\section{RESULTADOS E DISCUSSÕES}

Após a busca nas bases de dados, no período de 2015-2020, foram encontrados 539 artigos com as palavras-chave utilizadas, conforme o fluxograma apresentado na Figura 1. Após a avaliação dos artigos quanto aos critérios de inclusão e exclusão, foram eleitas 12 publicações para leitura na íntegra e desenvolvimento da discussão dos dados, as quais estão caracterizadas na Tabela 1.

Figura 1 - Fluxograma das etapas para selecionar as publicações utilizadas na revisão.

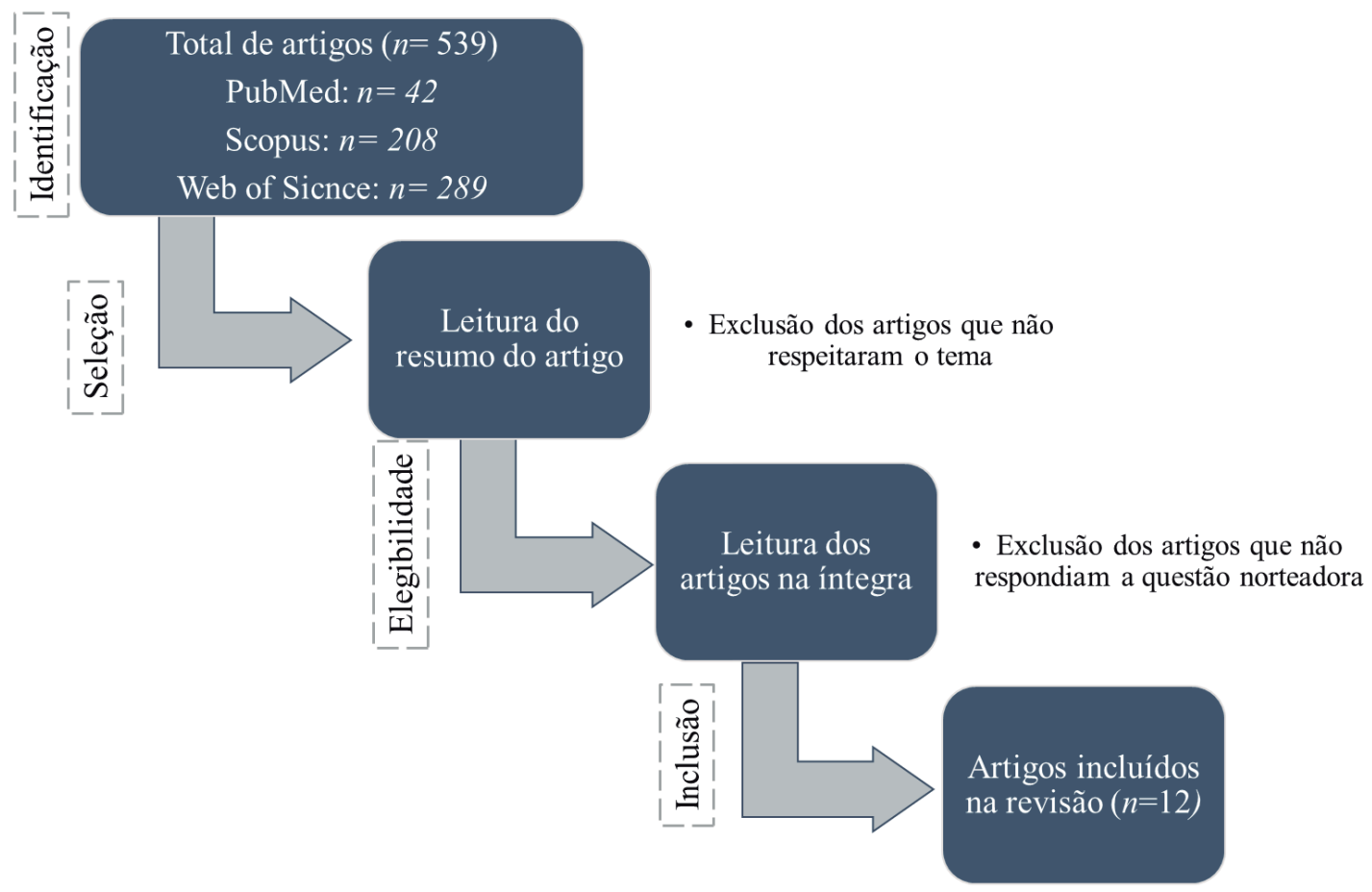

Fonte: Construção do autor.

Do total, seis publicações foram classificadas com nível de evidência II, tratando-se de estudos randomizados, duas publicações classificadas com nível de evidência III, estudos sem randomização, um estudo prospectivo, classificado como nível IV, e por fim, três publicações relacionadas a estudo de custo-efetividade, classificadas como nível V. Os resultados mostram que 50\% das publicações tratam da inserção do cetuximabe no protocolo FOLFOX, 33,3\% no protocolo FOLFIRI e $16,66 \%$ trazem os dois protocolos como comparação ao cetuximabe. Alguns dados dos estudos, como taxas de SLP, SG e TRO foram extraídos das publicações estão apresentados em tabelas (3 e 4), para uma melhor compreensão. 
Tabela 1 - Caracterização dos artigos elegidos para a revisão integrativa.

\begin{tabular}{ccccc}
\hline Autor & $\begin{array}{c}\text { Ano de } \\
\text { publicação }\end{array}$ & Periódico & Delineamento do estudo & $\begin{array}{c}\text { Nível de } \\
\text { evidência }\end{array}$ \\
\hline Bokemeyer et al. & 2015 & European Journal of Cancer & Estudo randomizado & II \\
\hline Soda et al. & 2015 & BMC Cancer & Estudo não randomizado & III \\
Martinelli et al. & 2016 & European Society for Medical Oncology & Estudo randomizado & II \\
\hline Kotake et al. & 2017 & Oncology Letters & Estudo prospectivo & IV \\
Qin et al. & 2018 & Journal of Clinical Oncology & Estudo randomizado & II \\
\hline Aranda et al. & 2018 & European Journal of Cancer & Estudo randomizado & II \\
Shankaran et al. & 2018 & American Journal of Clinical Oncology & Análise de custo-efetividade & V \\
\hline Price et al. & 2019 & Asia-Pacific Journal of Clinical Oncology & Estudo não randomizado & III \\
Bai et al. & 2019 & Cancer Management and Research & Análise de custo-efetividade & V \\
\hline Bridgewater et al. & 2020 & The Lancet. Oncology & Estudo randomizado & II \\
Han et al. & 2020 & Cancer control & Análise de custo-efetividade & V \\
\hline
\end{tabular}

Fonte: Construção do autor.

\section{ESQUEMA TERAPÊUTICO PARA O TRATAMENTO DO CCR}

A terapia padrão do adenocarcinoma colorretal por muitos anos foi o quimioterápico 5-fluorouracil (5-FU) que atua na inibição da síntese de DNA. Após estudos, ele foi combinado com o ácido folínico (Leucovorin ${ }^{\circledR}$ ) que age como modulador bioquímico, originando o protocolo denominado 5-FU/LV, melhorando a sobrevida dos pacientes quando comparado com a monoterapia de 5-FU. Esses esquemas terapêuticos variam na dose, no tempo de infusão e no tipo de infusão (contínua ou bolus) (DA SILVA et al., 2018; SAMA; COHEN, 2016; TONON; SECOLI; CAPONERO, 2007). Com o surgimento de um novo fármaco denominado irinotecano, foi criado um protocolo denominado IFL, no qual o irinotecano foi associado ao 5-FU/LV, mostrando melhoras nas taxas de resposta clínica. Em relação ao mecanismo de ação, o irinotecano e seu metabólito ativo SN-38 se ligam ao complexo DNA-topoisomerase I e impedem a religação das fitas únicas de DNA (CHABNER; LONGO, 2015). Em seguida, foi descoberta a oxaliplatina um agente quimioterápico com mecanismo de ação semelhante aos derivados da platina, que foi associado ao 5FU/LV infusional, originando o esquema FOLFOX (CHABNER; LONGO, 2015). Outras combinações com capecitabina mais oxaliplatina formam o esquema XELOX; já os regimes a base de irinotecano, fluorouracil e leucovorina compõem o esquema FOLFIRI; ou a associação do 5-FU, com oxaliplatina e irinotecano, chamado FOLFOXIRI (BRASIL, 2014; DA SILVA et al., 2018; SAMA; COHEN, 2016; SILVA; CARLOTTO; ROTTA, 2018). As doses dos principais protocolos utilizados estão apresentadas na Tabela 2. 
Tabela 2 - Doses usuais no tratamento de câncer colorretal metastático

\begin{tabular}{|c|c|}
\hline Protocolos de Tratamento & Exemplo de Doses usuais \\
\hline \multirow{2}{*}{ 5-FU/LV } & 5-Fluorouracil $\left(425 \mathrm{mg} / \mathrm{m}^{2}\right)$ - IV \\
\hline & Leucovorin $^{\circledR}\left(20 \mathrm{mg} / \mathrm{m}^{2}\right)$ - IV \\
\hline \multirow{4}{*}{ FOLFIRI } & 5-Fluorouracil $\left(400 \mathrm{mg} / \mathrm{m}^{2}\right)$ - IV bolus \\
\hline & 5-Fluorouracil $\left(2400 \mathrm{mg} / \mathrm{m}^{2}\right)$ - IV contínuo por 46 - $48 \mathrm{~h}$ \\
\hline & Leucovorin ${ }^{\circledR}\left(400 \mathrm{mg} / \mathrm{m}^{2}\right)$ - IV \\
\hline & Irinotecano $\left(180 \mathrm{mg} / \mathrm{m}^{2}\right)$ - IV \\
\hline \multirow{4}{*}{ FOLFOX-4 } & 5-Fluorouracil $\left(400 \mathrm{mg} / \mathrm{m}^{2}\right)$ - IV bolus \\
\hline & 5-Fluorouracil $\left(600 \mathrm{mg} / \mathrm{m}^{2}\right)$ - IV contínuo por $22 \mathrm{~h}$ \\
\hline & Leucovorin ${ }^{\circledR}\left(200 \mathrm{mg} / \mathrm{m}^{2}\right)$ - IV \\
\hline & Oxaliplatina $\left(85 \mathrm{mg} / \mathrm{m}^{2}\right)$ - IV \\
\hline \multirow{3}{*}{ FOLFOX-6/mFOLFOX-6 } & 5-Fluorouracil $\left(400 \mathrm{mg} / \mathrm{m}^{2}\right)$ - IV bolus \\
\hline & 5-Fluorouracil $\left(2400 \mathrm{mg} / \mathrm{m}^{2}\right)$ - IV contínuo por 46 - $48 \mathrm{~h}$ Leucovorin $^{\circledR}\left(400 \mathrm{mg} / \mathrm{m}^{2}\right)$ - IV \\
\hline & Oxaliplatina $\left(85 \mathrm{mg} / \mathrm{m}^{2}\right)$ - IV \\
\hline \multirow{4}{*}{ FOLFOXIRI } & 5-Fluorouracil (2400 - 3200mg/m²) - IV \\
\hline & Oxaliplatina $\left(85 \mathrm{mg} / \mathrm{m}^{2}\right)$ \\
\hline & Leucovorin ${ }^{\circledR}\left(400 \mathrm{mg} / \mathrm{m}^{2}\right)$ - IV \\
\hline & Irinotecano $\left(165 \mathrm{mg} / \mathrm{m}^{2}\right)$ - IV \\
\hline \multirow{3}{*}{ IFL } & Irinotecano $\left(100-125 \mathrm{mg} / \mathrm{m}^{2}\right)$ - IV \\
\hline & 5-Fluorouracil $\left(500 \mathrm{mg} / \mathrm{m}^{2}\right)$ - IV \\
\hline & Leucovorin $^{\circledR}\left(20 \mathrm{mg} / \mathrm{m}^{2}\right)$ - IV \\
\hline \multirow{2}{*}{ XELOX } & Oxaliplatina $\left(130 \mathrm{mg} / \mathrm{m}^{2}\right)$ - IV \\
\hline & Capecitabina $\left(2000 \mathrm{mg} / \mathrm{m}^{2}\right)$ - VO \\
\hline
\end{tabular}

\section{ONCOGÊNESE E CETUXIMABE - ASPECTOS GERAIS}

Com o avanço das pesquisas, os anticorpos monoclonais foram inseridos no tratamento antineoplásico, em monoterapia ou associados aos protocolos convencionais (YE et al., 2015). Os anticorpos monoclonais podem ser quiméricos, humanizados ou humanos. Os anticorpos quiméricos são formados por sequências de aminoácidos de origem humana, com sequências de origem murina nas regiões que se ligam aos epítopos antigênicos. Nos anticorpos humanizados, grande parte das sequências de aminoácidos é de origem humana, com uma parcela inferior correspondendo à origem murina, enquanto que os anticorpos humanos possuem somente sequências de origem humana (VIEIRA; SENA, 2009).

Os oncogenes originamse da desregulação de genes que fisiologicamente induzem a transcrição de proteínas associadas, que modulam o crescimento, diferenciação, apoptose e transdução de sinais intracelulares (proto-oncogenes). Os genes da família $R A S$ - RAt Sarcoma vírus (KRAS, NRAS e HRAS) são os oncogenes mais comuns nas neoplasias malignas e, quando mutados, levam à ativação permanente da proteína $R A S$ (LEAL et al., 2020). As mutações no $K R A S$ são mais frequentes em adenocarcinomas, enquanto no $N R A S$ são mais prevalentes em leucemias, carcinoma de tireoide e melanoma. As mutações no HRAS podem ser encontradas com maior frequência em carcinomas de bexiga e seminomas. Em condições normais, em que o gene $K R A S$ codifica a proteína em seu estado 
denominado selvagem ou "wildtype", a ligação de um fator de crescimento ao seu receptor de membrana (por exemplo o $E G F R$ ) ativa a proteína $R A S$. Isso desencadeia a ativação de vias de sinalização que culminam na indução de genes importantes na manutenção do fenótipo neoplásico. Na presença de determinadas mutações tumorais, em especial nos códons 12 e 13 do gene KRAS, ocorre ativação da proteína independentemente da presença do fator de crescimento. Dessa forma, a ocorrência dessas mutações passou a ser utilizada como biomarcador de resposta ao uso de anticorpos anti-EGFR, visto que o bloqueio do receptor passa a não ter efeito terapêutico, quando a proliferação celular está continuamente estimulada, devido à ativação constitutiva da via pela proteína $R A S$ que sofreu mutação (ADJEI, 2001; AMADO et al., 2008; GROTHEY; SARGENT, 2005).

O proto-oncogene humano BRAF (v-raf murine sarcoma viral oncogene homolog $B$ ), localizado no cromossomo 7 (7q34), codifica uma proteína de mesmo nome que está intrinsecamente envolvida na cascata de sinalização da MAP (mitogen-activated protein)/ERK (extracellular signal-regulated kinase) quinase. O BRAF é de fundamental importância para o adequado funcionamento celular, desde o seu crescimento, diferenciação, proliferação, senescência e até a apoptose. A via $R A S / R A F / M E K / E R K / M A P K$ está mutada em aproximadamente $30 \%$ de todos os tipos de câncer, sendo a mutação de $B R A F$ encontrada em quase $15 \%$ desse total, a qual também se expressa nos cânceres colorretais metastáticos, perfazendo 8 e $12 \%$ do total de casos notificados. Identifica-se que sua ocorrência é mutuamente excludente com mutações do gene $R A S$. Dessa forma, a presença da mutação $B R A F$ no câncer de colorretal metastático é considerada um biomarcador prognóstico negativo, pois está associado a pior prognóstico (LEAL et al., 2020).

$\mathrm{O}$ cetuximabe é um anticorpo monoclonal humano/murino quimérico IgG1 que se liga à porção extracelular do receptor EGFR (BASELGA, 2001). O EGFR é uma glicoproteína transmembrana que normalmente se expressa, em diversos tecidos humanos e está envolvida no controle do crescimento e da proliferação celular. Muitas neoplasias têm a expressão desregulada e aumentada do $E G F R$, resultando em proliferação, migração e adesão da célula tumoral, além do estímulo à angiogênese e inibição da apoptose. Tais alterações são observadas, principalmente, em neoplasias de cabeça e pescoço, cólon e pulmão e estão relacionadas com a progressão da doença, maior chance de metástase, redução da sobrevida e menor resposta à quimioterapia e radioterapia. A inibição desses receptores com agentes específicos como cetuximabe tem demonstrado eficácia no tratamento dessas neoplasias (RODARTE et al., 2009).

O cetuximabe é indicado no tratamento de câncer de colorretal metastático (CCRm) com expressão do EGFR e sem mutação do gene $R A S(K R A S$ e $N R A S)$ associado à quimioterapia, utilizado em combinação com FOLFIRI ou FOLFOX, ou como agente único em pacientes que tenham falhado à terapia baseada em oxaliplatina e irinotecano, ou que sejam intolerantes ao irinotecano (SAMA; COHEN, 2016). Para todas as indicações, cetuximabe deve ser administrado uma vez por semana. A dose inicial é de $400 \mathrm{mg}$ de cetuximabe por $\mathrm{m}^{2}$ de superfície corporal e todas as doses semanais subsequentes são de $250 \mathrm{mg} / \mathrm{m}^{2}$ (MERCK, 2020). 
Diferente dos efeitos adversos observados para os agentes antineoplásicos convencionais, tais como náuseas e queda de cabelo, a terapia com um inibidor do EGFR é uma terapia direcionada, portanto, tem efeitos colaterais relacionadas à infusão, as quais se manifestam geralmente durante a infusão inicial ou até horas após o término da mesma, podendo ocorrer em infusões subsequentes. Os principais efeitos relatados são distúrbios respiratórios, reações cutâneas, desequilíbrios eletrolíticos em que foram frequentemente observados níveis de magnésio no soro progressivamente decrescentes, que podem levar pacientes à hipomagnesemia grave; neutropenia e complicações infecciosas relacionadas e distúrbios cardiovasculares (BRASIL, 2013; MERCK, 2020).

\section{CETUXIMABE ASSOCIADO AOS PROTOCOLOS FOLFOX E FOLFIRI}

Uma vez que as mutações do gene $R A S$ nos códons 12 e 13 acarretam na falta de efetividade dos anticorpos anti-EGFR, Van Cutsem e colaboradores (2015) analisaram o efeito do tratamento de cetuximabe mais FOLFIRI em comparação com o FOLFIRI sozinho em pacientes com tumores positivos para mutações predefinidas em locos $R A S$ que não sejam os códons 12 ou 13 do KRAS. Também foi avaliado o efeito do tratamento em pacientes com tumores avaliáveis do tipo selvagem em todos os locos do KRAS. O estudo demonstrou resultados benéficos e significativos relacionados à associação de cetuximabe ao FOLFIRI no tratamento de pacientes com CCRm do tipo $R A S$ selvagem, como observado na Tabela 3. Para os pacientes com tumores portadores de mutações do $R A S$, nenhum benefício do tratamento com cetuximabe foi obtido (Tabela 3). Com os resultados deste estudo, pode-se concluir que de fato pacientes com mutações no gene $R A S$ não se beneficiam da terapia com cetuximabe e, além disso, ressalta a importância da pesquisa deste biomarcador para melhor decisão quanto ao regime terapêutico. No presente estudo foram observados eventos adversos (EAs) de grau $3 / 4$ (grave) e o mais comum na população do $R A S$ selvagem foi a neutropenia, que ocorreu em 30,9\% dos pacientes que receberam cetuximabe mais FOLFIRI e 20,1\% daqueles que receberam apenas FOLFIRI. Além disso, reações cutâneas, diarreia e reações relacionadas à infusão foram mais incidentes nos pacientes do cetuximabe mais FOLFIRI em comparação com o grupo isolado do FOLFIRI (VAN CUTSEM et al., 2015).

Corroborando com isso, esta mesma linha de pensamento foi abordada por Bokemeyer e colaboradores (2015), em que foi realizada uma reanálise dos resultados do estudo OPUS realizado inicialmente em 2011 (BOKEMEYER et al., 2011), avaliando adição do cetuximabe agora ao protocolo FOLFOX-4 em pacientes com tumores portadores de mutações em locos $R A S$ diferentes do códon 12 ou 13 do KRAS. O efeito do tratamento em pacientes com tumores do tipo selvagem em todos os locos $R A S$ também foi investigado. Devido ao tamanho limitado da população, a avaliação formal das interações com o tratamento de biomarcadores não foi possível e o estudo foi limitado a uma descrição dos resultados de eficácia nos desfechos para pacientes avaliáveis com tumores do tipo selvagem 
$K R A S$ exon-2 que abrigam outras mutações do $R A S$. Para a taxa de resposta objetiva e sobrevida livre de progressão, observou-se um possível benefício do cetuximabe mais FOLFOX-4 nos pacientes com tumores do tipo selvagem $R A S$ em comparação aos pacientes com tumores do tipo selvagem KRAS exon-2. Em pacientes com tumores do tipo RAS selvagem as taxas de SLP, SG e TRO foram significativamente superiores após administração do tratamento de cetuximabe mais FOLFOX-4 do que FOLFOX-4 sozinho (Tabela 3), em comparação aos pacientes $R A S$ mutado. Os resultados para a população do tipo selvagem do $R A S / B R A F$ foram muito semelhantes aos da população do tipo selvagem do $K R A S$. Também foi investigado os resultados para o grupo de pacientes com qualquer mutação $K R A S$. Entre os desfechos de eficácia, um evidente efeito prejudicial associado à adição de cetuximabe ao FOLFOX-4 foi aparente nessa população, indicando mais uma vez que a presença de qualquer mutação do gene $R A S$ torna a terapia com cetuximabe ineficaz (BOKEMEYER et al., 2015).

Tabela 3 - Dados de sobrevida livre de progressão (SLP), sobrevida global (SG) e taxa de resposta objetiva (TRO) do estudo de Eric Van Cutsem e colaboradores (2015), Bokemeyer e colaboradores (2015), Qin e colaboradores (2018) e Bridgewater e colaboradores (2020).

\begin{tabular}{|c|c|c|c|c|}
\hline \multicolumn{5}{|c|}{ Eric Van Cutsem e colaboradores (2015) } \\
\hline & \multicolumn{2}{|c|}{ RAS SELVAGEM } & \multicolumn{2}{|c|}{ RAS MUTANTE } \\
\hline & CETUXIMABE & \multirow{2}{*}{ FOLFIRI } & CETUXIMABE & \multirow{2}{*}{ FOLFIRI } \\
\hline & E FOLFIRI & & E FOLFIRI & \\
\hline SLP (meses) & 11,4 & 8,4 & 7,2 & 6,9 \\
\hline SG (meses) & 28,4 & 20,2 & 18,2 & 20,7 \\
\hline TRO (\%) & 66,3 & 38,6 & 34,4 & 35,5 \\
\hline \multicolumn{5}{|c|}{ Bokemeyer e colaboradores (2015) - OPUS } \\
\hline & \multicolumn{2}{|c|}{ RAS SELVAGEM } & \multicolumn{2}{|c|}{ RAS MUTANTE } \\
\hline & CETUXIMABE E & & CETUXIMABE E & \\
\hline & FOLFOX-4 & FOLFOX-4 & FOLFOX-4 & FOLFOX-4 \\
\hline SLP (meses) & 12,0 & 5,8 & 8,3 & 6,9 \\
\hline SG (meses) & 19,8 & 17,8 & 19,5 & 17,8 \\
\hline TRO $(\%)$ & 58,0 & 29,0 & 57,0 & 32,0 \\
\hline \multicolumn{5}{|c|}{ Qin e colaboradores (2018) - TAILOR } \\
\hline & \multicolumn{4}{|c|}{ RAS SELVAGEM } \\
\hline & CETUXIMA & OLFOX-4 & \multicolumn{2}{|c|}{ FOLFOX-4 } \\
\hline SLP (meses) & & & \multicolumn{2}{|c|}{7,4} \\
\hline SG (meses) & & & \multicolumn{2}{|c|}{17,8} \\
\hline TRO $(\%)$ & & & \multicolumn{2}{|c|}{39,5} \\
\hline \multicolumn{5}{|c|}{ Bridgewater e colaboradores (2020) - EPOC } \\
\hline & \multicolumn{4}{|c|}{ KRAS SELVAGEM } \\
\hline & CETUXIM & FOLFOX, & \multirow{2}{*}{\multicolumn{2}{|c|}{ FOLFOX, FOLFIRI E CAPOX }} \\
\hline & FOLFIR & $\mathrm{POX}$ & & \\
\hline SLP (meses) & & & \multicolumn{2}{|c|}{22,2} \\
\hline SG (meses) & & & \multicolumn{2}{|c|}{81,0} \\
\hline TRO $(\%)$ & & & \multicolumn{2}{|c|}{61,0} \\
\hline
\end{tabular}

Fonte: Construção do autor. 
A eficácia e segurança da administração quinzenal de cetuximabe e mFOLFOX-6 ou XELOX como quimioterapia de primeira linha em pacientes com câncer colorretal metastático foi avaliada no estudo FLEET em 2015, realizado no Japão. De toda a população envolvida no estudo, sessenta e dois pacientes com câncer colorretal metastático de tipo selvagem KRAS (códons 12,13 e 61)/BRAF (V600E) não tratado anteriormente participaram do estudo. Os pacientes receberam cetuximabe mais mFOLFOX-6 (FOLFOX + Cmab) ou cetuximabe mais XELOX (XELOX + Cmab), a cada quinze dias. O tratamento foi mantido até a progressão da doença ou o aparecimento de toxicidade intolerável. O endpoint primário foi a taxa de resposta, enquanto endpoints secundários foram sobrevida livre de progressão, sobrevida global, taxa de controle da doença, intensidade da dose, taxa de conversão para ressecção cirúrgica e segurança. A taxa de resposta foi de 64,9\% para pacientes que receberam FOLFOX + Cmab e 72,0\% para pacientes em tratamento com XELOX + Cmab. A SLP foi semelhante para os dois grupos, na faixa de 13 meses e a média de SG foi superior no grupo que recebeu XELOX + Cmab (47 meses versus 38,1 meses). O evento adverso de grau 3/4 mais comum foi neutropenia, seguido por erupção acneiforme e paroníquia. Foi observado também erupção cutânea em alguns pacientes, devido a administração do cetuximabe. Os autores ainda ressaltam que nenhum paciente foi a óbito após 28 dias do último ciclo de tratamento. Apesar do número pequeno de pacientes envolvidos neste estudo, ele traz dados promissores em relação ao uso do protocolo XELOX juntamente com o cetuximabe, mostrando ser eficaz e com efeitos adversos toleráveis (SODA et al., 2015).

A combinação do protocolo FOLFIRI mais cetuximabe teve sua segurança e eficácia avaliadas em subgrupos definidos por idade, para o tratamento de primeira linha do CCRm do tipo KRAS (exon-2) selvagem. Foram utilizados três pontos de corte etários para definir a população idosa ( $\geq 65, \geq 70$ e $\geq 75$ anos). A análise de sequenciamento identificou um subgrupo de pacientes quádruplos para os genes $K R A S, N R A S$, $B R A F, P I K 3 C A$ do tipo selvagem, onde evidenciou-se um melhor resultado em comparação com pacientes portadores de mutações nesses mesmos genes. A TRO não foi significativamente diferente entre os subgrupos etários nas três populações e o tratamento com FOLFIRI mais cetuximabe resultou em uma eficácia comparável na SLP nos subgrupos etários da população com intenção de tratar. No presente estudo foi observado EAs de todos os graus, e a incidência foi semelhante em pacientes mais jovens e idosos. A neutropenia foi o EA hematológico mais prevalente. No entanto, pacientes com idade $\geq 75$ anos apresentaram maior taxa de neutropenia grau 3/4 em comparação com pacientes com idade $<75$ anos. Todos os graus de EAs não hematológicos foram relatados como semelhantes nos três grupos etários. Náuseas e vômitos foram os EAs mais relatados na população geral e nenhuma diferença significativa foi observada entre as faixas etárias. As reações cutâneas foram comparáveis entre os três subgrupos e nenhuma diferença foi observada nas toxicidades de grau 3/4. Já os pacientes com idade $\geq 75$ anos foi observada uma diferença significativa nas taxas de diarreia de grau 3/4 em comparação com pacientes com menos de 75 anos. Taxas de fadiga de grau 3/4 significativamente mais altas foram relatadas em pacientes $<75$ anos em comparação com a população idosa (MARTINELLI et al., 2016). 
O estudo CELINE avaliou a eficácia e segurança da administração quinzenal de cetuximabe em combinação com FOLFOX-6 no tratamento de primeira linha do CCRm do tipo KRAS selvagem. Acredita-se que propor um regime de administração quinzenal pode ser mais conveniente e melhorar significativamente a qualidade de vida do paciente. O presente estudo fez uma análise de braço único, onde os pacientes receberam apenas o cetuximabe mais FOLFOX6 como tratamento quimioterápico. Obteve-se uma taxa de resposta objetiva de $70 \%$ ao tratamento. Além disso, os pacientes foram avaliados de acordo com o órgão metastático, resultando em $72 \%$ a taxa de resposta em pacientes com metástases hepáticas, 73,3\% em pacientes com metástases pulmonares e $63,2 \%$ em pacientes com metástase linfática, sendo assim, não foi observado diferenças na taxa de resposta objetiva entre diferentes órgãos afetados por metástases. Portanto, a taxa de controle da doença foi de 95\%, demonstrando a eficácia do tratamento. Os pacientes tiveram uma sobrevida livre de progressão mediana de 13,8 meses e uma sobrevida global mediana de 31,0 meses. Diante disso, 13 pacientes $(21,7 \%)$ foram submetidos à cirurgia para a retirada de metástases hepáticas, e 10 pacientes submetidos à ressecção cirúrgica completa (R0). Efeitos adversos de qualquer grau ocorreram em todos os pacientes e EAs de grau 3/4 foram observados em 66,7 \% dos pacientes, sendo os mais comuns a leucopenia (41,7\%) e a paroníquia (18,3\%). De todos os pacientes com grau 3/4 de EAs, 8,3\% apresentaram erupção cutânea semelhante à acne e um paciente apresentou reações relacionadas à infusão. O presente estudo demonstrou a eficácia e segurança equivalentes da administração quinzenal do cetuximabe em combinação com o FOLFOX-6 em pacientes com CCRm do tipo selvagem $K R A S$, em comparação com o regime de administração semanal. Com isso, há uma redução na frequência de visitas ao hospital e consequentemente uma melhora na qualidade de vida dos pacientes. Além disso, o regime de combinação quinzenal pode simplificar a administração do tratamento para os profissionais de saúde e ser mais econômico (KOTAKE et al., 2017).

O estudo TAILOR foi um ensaio clínico de fase III, que avaliou especificamente pacientes com CCRm cujo tumores eram tipo $R A S$ selvagem (KRAS/NRAS, éxons 2 a 4). Para os pacientes que receberam o tratamento quimioterápico de cetuximabe mais FOLFOX-4, observou-se uma melhora significativa no tempo de SLP, SG e TRO (Tabela 2). Finalmente, sete de oito pacientes do braço cetuximabe mais FOLFOX-4 e dois de seis pacientes do braço FOLFOX-4, submetidos à cirurgia com intenção curativa, tiveram ressecções R0. Neste mesmo estudo também foi analisado o status de mutação tumoral do gene BRAF do tipo selvagem ou mutante. A adição do cetuximabe mais FOLFOX-4 em pacientes do gene $K R A S / B R A F$ selvagem foi benéfico em relação ao tempo de SLP (mediana de 9,3 versus 7,5 meses). Por outro lado, em pacientes do gene $K R A S / B R A F$ mutado não demonstrou benefício no tempo de SLP em comparação aos pacientes que receberam FOLFOX-4 sozinho (mediana de 2,0 versus 3,8 meses, respectivamente). O estudo também relatou os EAs no tratamento da população com tumores $R A S$ selvagem. A neutropenia de grau $\geq 3$ foi o EA mais frequente no tratamento 
de ambos os braços. Ocorreram reações cutâneas de grau $\geq 3$ em 25,8\% dos pacientes no braço de cetuximabe mais FOLFOX-4. Foram detectados EAs graves por 19,1\% dos pacientes que receberam cetuximabe mais FOLFOX-4 em comparação com 13,1\% dos pacientes tratados com FOLFOX-4 (QIN et al., 2018).

O estudo MACRO02 TTD, realizado em 2018, foi um estudo multicêntrico, randomizado e de fase II que avaliou mFOLFOX+ cetuximabe seguido por mFOLFOX + cetuximabe de manutenção ou cetuximabe como agente único em pacientes com câncer colorretal metastático. Os pacientes com mCRC não tratados, com KRAS do tipo selvagem, foram selecionados para receber mFOLFOX-6 + cetuximabe (8 ciclos por 2 semanas), seguido de terapia de manutenção, sendo divididos em dois grupos: cetuximabe como agente único (Braço-A) ou mFOLFOX-6 + cetuximabe (Braço-B) até a progressão. O desfecho primário foi a sobrevida livre de progressão em 9 meses. Não houve diferença significativa de SLP ou SG nos dois grupos, bem como a TRO foi semelhante para ambos os tratamentos. O perfil de segurança foi semelhante entre os braços, e todos os pacientes experimentaram pelo menos um evento adverso, sendo os mais comuns neutropenia, erupção cutânea acneiforme e neuropatia sensorial, em qualquer grupo. $\mathrm{O}$ estudo sugere que a terapia de manutenção utilizando cetuximabe como agente único pode ser uma opção vantajosa frente a associação com quimioterapia (ARANDA et al., 2018).

A localização do tumor primário no trato colorretal (direita versus esquerda) tem um valor prognóstico significativo para a sobrevida do paciente. De fato, pacientes com tumores $R A S$ selvagem do lado direito apresentam um pior prognóstico, isto é, resultados de sobrevida muito inferior aos pacientes com tumores do lado esquerdo, independentemente do tratamento recebido. Além disso, o lado direito parece ter valor preditivo, uma vez que pacientes com CCRm do tipo $R A S$ selvagem do lado direito têm demonstrado menos benefícios no tratamento com cetuximabe do que pacientes com RAS selvagem do lado esquerdo (PRICE et al., 2019).

Tal questão foi investigada no estudo APEC, o qual demonstrou que o cetuximabe de primeira linha com administração quinzenal em combinação com FOLFOX ou FOLFIRI produziu boa resposta e aumento da sobrevida em uma população de pacientes com CCRm do tipo RAS selvagem. Além disso, o estudo não encontrou evidências de diferenças na eficácia entre a quimioterapia com FOLFIRI e FOLFOX quando combinada com cetuximabe (Tabela 4). Poucos pacientes no estudo APEC foram submetidos à cirurgia para doença metastática, mas as taxas de ressecção foram semelhantes entre os subgrupos do lado esquerdo e do lado direito (10,8 \% e 10,3\%, respectivamente). O estudo não revelou novos achados de segurança para quimioterapia de primeira linha com cetuximabe mais FOLFOX ou FOLFIRI, e não há evidências de que o perfil de segurança seja significativamente diferente entre direita e esquerda no CCRm (PRICE et al., 2019). 
Tabela 4 - Valores de sobrevida livre de progressão (SLP), sobrevida global (SG) e taxa de resposta objetiva (TRO) para pacientes com tumores do lado direito versus lado esquerdo. Ambos receberam tratamento com cetuximabe mais FOLFOX ou FOLFIRI, quando participaram do estudo APEC (Price et al., 2019).

\begin{tabular}{ccccc}
\hline \multicolumn{5}{c}{ RAS SELVAGEM } \\
\hline & LADO DIREITO & \multicolumn{2}{c}{ LADO ESQUERDO } \\
\hline & CETUXIMABE E & CETUXIMABE E & CETUXIMABE E & CETUXIMABE E \\
& FOLFOX & FOLFIRI & FOLFOX & FOLFIRI \\
SLP (meses) & 8,3 & 15,4 & 14,2 & 12,8 \\
SG (meses) & 21,8 & 32,1 & 30,6 & 31,7 \\
TRO (\%) & 52,6 & 50,0 & 65,5 & 74,4 \\
\hline
\end{tabular}

Fonte: Construção do autor.

No novo estudo EPOC, foi avaliado o benefício da adição de cetuximabe à quimioterapia sistêmica perioperatória (antes e após a cirurgia) em pacientes com CCR e metástase hepática ressecável com KRAS do tipo selvagem (códons 12, 13 e 61). Os pacientes foram aleatoriamente designados para receber quimioterapia com ou sem cetuximabe antes e após a ressecção hepática. A quimioterapia consistiu em três regimes terapêuticos diferentes: FOLFOX, FOLFIRI e CAPOX (capecitabina e oxaliplatina). Surpreendentemente, essa adição resultou em uma sobrevida livre de progressão mais curta nos pacientes tratados com cetuximabe (Tabela 3). Não houve diferença significativa na proporção de pacientes que obtiveram uma resposta entre os grupos de tratamento. A maioria dos pacientes submetidos à operação teve uma ressecção R0, sendo $82 \%$ dos pacientes do grupo de quimioterapia isolada e $79 \%$ dos pacientes do grupo de quimioterapia mais cetuximabe. A sobrevida pós-progressão mediana foi de 33,5 meses no grupo de quimioterapia sozinha em comparação com 23,5 meses no grupo de quimioterapia mais cetuximabe. Os EAs de grau 3/4 mais comuns foram à diminuição da contagem de neutrófilos (19\% no grupo de quimioterapia per se versus $15 \%$ no grupo de quimioterapia mais cetuximabe), diarreia (10\% versus $10 \%$ ), erupção cutânea (1\% versus $16 \%$ ), eventos tromboembólicos ( $7 \%$ versus $8 \%$ ), letargia ( $7 \%$ versus $7 \%$ ), mucosite oral ( $2 \%$ versus $10 \%)$, vômitos ( $5 \%$ versus $5 \%$ ), neuropatia periférica ( $6 \%$ versus $4 \%$ ) e dor ( $4 \%$ versus $4 \%)$. No geral, o estudo sugere que a sobrevida em pacientes com metástases hepáticas colorretais operáveis é significativamente pior com o uso de cetuximabe em combinação com quimioterapia no período perioperatório do que apenas com quimioterapia. Cetuximabe não deve ser usado como terapia neoadjuvante em pacientes com metástases hepáticas colorretais operáveis (BRIDGEWATER et al., 2020)

Por fim, é de suma importância analisar os custos associados ao tratamento quando se utiliza anticorpos monoclonais. Por serem medicamentos biológicos, os mesmos têm valor elevados para produção e por isso acabam tornando os tratamentos onerosos. O estudo FIRE-3 realizado nos Estados Unidos da América (EUA) com pacientes com CCRm $R A S$ selvagem, que receberam como tratamento de primeira linha cetuximabe mais FOLFIRI ou bevacizumabe mais FOLFIRI, avaliou o custo-benefício dos anticorpos monoclonais. Os resultados demonstraram que o protocolo composto por cetuximabe pareceu melhorar os resultados de saúde e usar recursos financeiros de forma mais 
eficiente do que FOLFIRI mais bevacizumabe (SHANKARAN et al., 2018). Essas mesmas conclusões foram obtidas pelo estudo de Han e colaboradores (2020), que investigaram a relação custo-benefício ao tratar pacientes com FOLFIRI mais cetuximabe ou bevacizumabe da perspectiva do sistema de saúde chinês (HAN et al., 2020). Ainda, foi realizada uma análise de custo-efetividade sobre a associação do cetuximabe no protocolo FOLFOX por pesquisadores chineses. A relação custo-efetividade incremental (RCEI) foi o desfecho primário de saúde, o qual foi associado aos custos e aos anos de vida ajustados pela qualidade $(Q A L Y)$. Na perspectiva da comunidade chinesa, a combinação de cetuximabe e FOLFOX-4 não é uma estratégia econômica em comparação com FOLFOX-4 sozinho para o tratamento de primeira linha de pacientes com CCRm do tipo RAS selvagem (BAI et al., 2019).

No Brasil, o relatório da Comissão Nacional de Incorporação de Tecnologias - CONITEC, publicado em 2018, mostra uma avaliação econômica para adição de cetuximabe à quimioterapia em pacientes com CCRm RAS selvagem com doença limitada ao fígado, em primeira linha de tratamento, o qual apresentou RCEI de R\$ 56.750,00 por ano de vida ganho quando comparado à quimioterapia isolada. A análise de impacto orçamentário estimou um valor acumulado superior a R $\$ 326$ milhões em cinco anos, após a inclusão de cetuximabe. A análise de sensibilidade demonstrou que esse valor pode variar de R 153 milhões a R \$ 491 milhões em cinco anos. A decisão do CONITEC foi de não incorporar o cetuximabe para primeira linha para pacientes com câncer colorretal metastático (CCRm) RAS selvagem, com doença limitada ao fígado, em combinação com os regimes quimioterápicos FOLFIRI ou FOLFOX, no âmbito do Sistema Único de Saúde - SUS, dada pela Portaria no 4, publicada no DOU n ${ }^{\circ} 18$, do dia 25 de janeiro de 2018, seção 1, pág. 123. Entretanto, tal decisão não inviabiliza o uso do medicamento pelos usuários do SUS. As Diretrizes Diagnósticas e Terapêuticas do Câncer do Cólon e Reto, do Ministério da Saúde, recomendam o tratamento quimioterápico local ou sistêmico. A definição do esquema de tratamento deve ser definida pelo médico em conjunto com o paciente, desde que seu valor esteja enquadrado ao valor de reembolso proposto para as Autorizações de Procedimentos de Alta Complexidade (APAC) atualmente disponíveis no SUS para o tratamento do CCRm. Desta forma, assumiu-se como os protocolos quimioterápicos padrões de tratamento FOLFIRI ou FOLFOX, sendo cetuximabe proposto como intervenção add-on aos esquemas previamente mencionados (BRASIL, 2014, 2018).

\section{CONCLUSÕES}

Considerando os trabalhos abordados nesta revisão integrativa de literatura, a inclusão do cetuximabe no tratamento de primeira linha do câncer colorretal metastático promove uma melhora em relação aos esquemas padrões de quimioterapia. Apesar de alguns estudos não demonstrarem diferenças significativas entre quimioterapia e quimioterapia associada ao cetuximabe em alguns parâmetros, pode-se concluir que de uma maneira geral a inserção do anticorpo monoclonal anti-EGFR nos 
protocolos FOLFIRI e FOLFOX traz benefícios para a maioria dos pacientes. Além disso, observa-se que marcadores como a mutação no gene $K R A S$ e $B R A F$ são essenciais para escolha do cetuximabe no câncer colorretal, pois na maioria das vezes a mutação desse gene não é um fator de bom prognóstico. Vale ressaltar que a administração do cetuximabe causa reações infusionais e, por isso, é de extrema relevância os cuidados da equipe multiprofissional durante a infusão do medicamento nos centros oncológicos e hospitais. Por fim, é válido exaltar a importância e o impacto dos estudos de revisão, os quais trazem informações que ajudam a nortear abordagens terapêuticas e contribuem na atuação conjunta da Equipe Multiprofissional em Terapia Antineoplásica (EMTA) para proporcionar um tratamento seguro e eficaz para os pacientes.

\section{CONFLITOS DE INTERESSE}

Os autores declaram não haver conflitos de interesse.

\section{FINANCIAMENTO}

Nenhum financiamento ou apoio foi recebido para o trabalho.

\section{REFERÊNCIAS}

ADAM, R. et al. Patients With Initially Unresectable Colorectal Liver Metastases: Is There a Possibility of Cure? Journal of Clinical Oncology, v. 27, n. 11, p. 1829-1835, 2009.

ADJEI, A. A. Blocking Oncogenic Ras Signaling for Cancer Therapy. Journal of the National Cancer Institute, v. 93, n. 14, p. 1062-1074, 2001.

AMADO, R. G. et al. Wild-Type KRAS Is Required for Panitumumab Efficacy in Patients With Metastatic Colorectal Cancer. Journal of Clinical Oncology, v. 26, n. 10, p. 1626-1634, 2008.

ARANDA, E. et al. First-line mFOLFOX plus cetuximab followed by mFOLFOX plus cetuximab or single-agent cetuximab as maintenance therapy in patients with metastatic colorectal cancer: Phase II randomised MACRO2 TTD study. European Journal of Cancer, v. 101, p. 263-272, 2018.

BAI, L. et al. Cost-Effectiveness Analysis of First-Line Cetuximab Plus Leucovorin, Fluorouracil, and Oxaliplatin ( FOLFOX-4) versus FOLFOX-4 in Patients with RAS Wild-Type Metastatic Colorectal Cancer. Cancer Management and Research, v. 11, n. 12, p. 10419-10426, 2019. 
BASELGA, J. The EGFR as a target for anticancer therapy-focus on cetuximab. European Journal of Cancer, v. 37, n. SUPPL. 4, p. 16-22, 2001.

BOKEMEYER, C. et al. Efficacy according to biomarker status of cetuximab plus FOLFOX-4 as first-line treatment for metastatic colorectal cancer: the OPUS study. Annals of Oncology, v. 22, n. 7 , p. 1535-1546, 2011.

BOKEMEYER, C. et al. FOLFOX4 plus cetuximab treatment and RAS mutations in colorectal cancer. European Journal of Cancer, v. 51, n. 10, p. 1243-1252, 2015.

BRASIL. Cetuximabe em combinação com FOLFIRI ou FOLFOX no tratamento do câncer colorretal metastático KRAS selvagem com metástases hepáticas exclusivas. Ministério da Saúde. Secretaria de Ciência, Tecnologia e Insumos Estratégicos. Departamento de Gestão e Incorporação de Tecnologias em Saúde, 2013.

BRASIL. Protocolos clínicos e diretrizes terapêuticas em oncologia. Secretaria de Atenção à Saúde - Ministério Da Saúde, v. 1, p. 356, 2014.

BRASIL. Cetuximabe para o tratamento do câncer colorretal metastático RAS selvagem com doença limitada ao fígado em primeira linha. Ministério da Saúde - Secretaria de Ciência, Tecnologia e Insumos Estratégicos - Departamento de Gestão e Incorporação de Tecnologias em Saúde, 2018.

BRIDGEWATER, J. A. et al. Systemic chemotherapy with or without cetuximab in patients with resectable colorectal liver metastasis (New EPOC): long-term results of a multicentre, randomised, controlled, phase 3 trial. The Lancet Oncology, v. 21, n. 3, p. 398-411, 2020.

CHABNER, B. A.; LONGO, D. L. Manual de oncologia de Harrison. 2 edição ed. [s.n.].

DA SILVA, W. C. et al. Comparative Effectiveness and Safety of Monoclonal Antibodies (Bevacizumab, Cetuximab, and Panitumumab) in Combination with Chemotherapy for Metastatic Colorectal Cancer: A Systematic Review and Meta-Analysis. BioDrugs, v. 32, n. 6, p. 585-606, 2018.

DE CAMPOS, F. G. C. M. et al. Incidência de câncer colorretal em pacientes jovens. Revista do Colegio Brasileiro de Cirurgioes. Revista do Colegio Brasileiro de Cirurgioes, v. 44, n. 2, 208-215, 2017. 
DEKKER, E. et al. Colorectal cancer. The Lancet, v. 394, n. 10207, p. 1467-1480, 2019.

GROTHEY, A.; SARGENT, D. Overall Survival of Patients With Advanced Colorectal Cancer Correlates With Availability of Fluorouracil, Irinotecan, and Oxaliplatin Regardless of Whether Doublet or Single-Agent Therapy Is Used First Line. Journal of Clinical Oncology, v. 23, n. 36, p. 9441-9442, 2005.

HAN, J. et al. Cost-Effectiveness Analysis of First-Line FOLFIRI Combined With Cetuximab or Bevacizumab in Patients With RAS Wild-Type Left-Sided Metastatic Colorectal Cancer. Cancer Control, v. 27, n. 1, 2020.

INCA. Tipos de câncer. Disponível em: https://bit.ly/3bn4NGV. Acesso em: 30 set. 2019.

KOTAKE, M. et al. Multicenter phase II study of infusional 5-fluorouracil (5-FU), leucovorin, and oxaliplatin, plus biweekly cetuximab as first-line treatment in patients with metastatic colorectal cancer (CELINE trial). Oncology Letters, v. 13, n. 2, p. 747-753, 2017.

LEAL, A. Et al. Biomarcadores em Oncologia. Editora Manole Ltda. por meio de contrato de coedição com a AEON - Assessoria Especializada em Oncologia Ltda, v. 1 edição, p. 256, 2020.

MARTINELLI, E. et al. Clinical activity and tolerability of FOLFIRI and cetuximab in elderly patients with metastatic colorectal cancer in the CAPRI-GOIM first-line trial. European Society for Medical Oncology, v. 1, n. 6, p. e000086, 2016.

MELNYK, B. M.; FONEOUT-OVERHOLT, E. Making the case for evidence-based practice. In: Evidence-Based Practice in Nursing \& Healthcare: A Guide to Best Practice. [s.n.]. p. 3-24.

MENDES, K. D. S.; SILVEIRA, R. C. DE C. P.; GALVÃO, C. M. Revisão integrativa: método de pesquisa para a incorporação de evidências na saúde e na enfermagem. Texto \& Contexto - Enfermagem, v. 17, n. 4, p. 758-764, 2008.

MERCK S.A. Erbitux - Bula atualizada 06/01/2020. ANVISA, 2020. 
PRICE, T. et al. Phase II APEC trial : The impact of primary tumor side on outcomes of first-line cetuximab plus FOLFOX or FOLFIRI in patients with RAS wild-type metastatic colorectal cancer. Asia-Pacific Journal of Clinical Oncology, v. 15, n. 4, p. 225-230, 2019.

QIN, S. et al. Efficacy and Tolerability of First-Line Cetuximab Plus Leucovorin, Fluorouracil, and Oxaliplatin (FOLFOX-4) Versus FOLFOX-4 in Patients With RAS Wild-Type Metastatic Colorectal Cancer: The Open-Label, Randomized, Phase III TAILOR Trial. Journal of Clinical Oncology, v. 36, n. 30, p. 3031-3039, 2018.

RODARTE, C. M. et al. Reações cutâneas secundárias ao uso dos inibidores do receptor de fator de crescimento epidérmico: Relato de dois casos. Anais Brasileiros de Dermatologia, v. 84, n. 6, p. 667-670, 2009.

SAMA, A. R.; COHEN, S. J. Front-Line Treatment of Metastatic Colorectal Cancer. Journal of Oncology Practice, v. 12, n. 12, p. 1231-1233, 2016.

SANTOS JR., J. C. M. Câncer ano-reto-cólico: aspectos atuais II - câncer colorretal - fatores de riscos e prevenção. Revista Brasileira de Coloproctologia, v. 27, n. 4, p. 459-473, 2007.

SHANKARAN, V. et al. Cost-Effectiveness of Cetuximab as First-line Treatment for Metastatic Colorectal Cancer in the United States. American Journal of Clinical Oncology, v. 41, n. 1, p. 65-72, 2018.

SILVA, A. A. DA; CARLOTTO, J.; ROTTA, I. Standardization of the infusion sequence of antineoplastic drugs used in the treatment of breast and colorectal cancers. Einstein (Sao Paulo), v. 16, n.2, p. 1-9, 2018.

SODA, H. et al. Multicenter Phase II study of FOLFOX or biweekly XELOX and Erbitux (cetuximab) as first-line therapy in patients with wild-type KRAS / BRAF metastatic colorectal cancer: The FLEET study. BMC Cancer, v. 15, p. 1-10, 2015.

TONON, L. M.; SECOLI, S. R.; CAPONERO, R. Câncer colorretal : uma revisão da abordagem terapêutica com bevacizumabe. Revista Brasileira de Cancerologia, v. 53, n. 2, p. 173-182, 2007.

VAN CUTSEM, E. et al. Fluorouracil, leucovorin, and irinotecan plus cetuximab treatment and RAS mutations in colorectal cancer. Journal of Clinical Oncology, v. 33, n. 7, p. 692-700, 2015. 
VIEIRA, F. M. A. C. AND SENA, V. O. D. Artigo de Revisão Monoclonal therapy in metastatic colorectal cancer. ABCD. Arquivos Brasileiros de Cirurgia Digestiva (São Paulo), v. 22, n. 1, p. 45-49, 2009.

YE, L. et al. Impact of early tumor shrinkage on clinical outcome in wild-type-KRAS colorectal liver metastases treated with cetuximab. Journal of Gastroenterology and Hepatology, v. 30, n. 4, p. 674-679, 2015. 